greater number of diagnostic outcomes would produce wider confidence limits for the kappa statistics if the population size remained the same. ${ }^{9}$ It therefore seems than the kappa statistic is not a sensitive indicator of performance in cytopathological or histopathological quality assurances schemes unless large numbers of samples are used. Other indicators should be considered, possibly using systems which weight the outcomes according to the importance of the result in clinical practice. ${ }^{10}$ The present study did not include the function of time and the persistence of low kappa statistics over a number of cycles of a quality assurance scheme would be a more reliable indicator of possible unsatisfactory performance. However, the frequency of cycles in most schemes is such that at least two years would elapse before such an assessment would be possible.

1 Landis JR, Koch GG. The measurement of observer agreement for categorical data. Biometrics 1977;33:159-74.

2 Silcocks PB. Some issues in observer error studies in pathology. F Pathol 1992;168:255-6.

3 Svanholm H, Starklint H, Gundersen HJ, Fabricius J, Barlebo H, Olsen S. Reproducibility of histomorphologic Barlebo $\mathrm{H}$, Olsen $\mathrm{S}$. Reproducibility of histomorphologic diagnoses with special refe

4 Donker DK, Hasman A, van Geijn HP. Interpretation of low kappa values. Int $\mathcal{F}$ Biomed Comput 1993;33:55-64.

5 Silcocks PB. Measuring repeatability and validity of histological diagnosis - a brief review with some practica examples. $\mathcal{F}$ Clin Pathol 1983;36:1269-75.

6 Cytology Sub-Group of the National Coordinating Committee for Breast Screening Pathology. Guidelines for Cytology Procedures and Reporting in Breast Cancer Cytology Procedures and Reporting in Breast Cance BSP Publications, 1993 .

7 Seldenrijk CA, Morson BC, Meuwissen SG, Schipper NW, Lindeman J, Meijer CJ. Histopathological evaluation of colonic mucosal biopsy specimens in chronic inflammatory bowel disease: diagnostic implications. Gut 1991, 32:1514-20

8 Andrew A, Wyatt JI, Dixon MF. Observer variation in the assessment of chronic gastritis according to the Sydney system. Histopathology 1994;25:317-22.

9 Morris JA. Information and observer disagreement in histopathology. Histopathology 1994;25:123-8.
10 Furness PN, Lauder I. Response analysis in histopathology EQA schemes. F Clin Pathol 1993;46:357-63.

\section{Appendix}

\begin{tabular}{llll}
\hline & Diagnosis & & \\
\cline { 2 - 4 } Outcome & Benign & Malignant & Total \\
\hline Benign & $\boldsymbol{a}$ & $\boldsymbol{b}$ & $\boldsymbol{g}$ \\
Malignant & $\boldsymbol{c}$ & $\boldsymbol{d}$ & $\boldsymbol{h}$ \\
Total & $\boldsymbol{e}$ & $\boldsymbol{f}$ & $\boldsymbol{i}$ \\
\hline
\end{tabular}

In the initial state of the model $\boldsymbol{b}=0$ and $c=0$, and the rest of the cases are spilt between $\boldsymbol{a}$ and $\boldsymbol{d}$ in the proportions for that particular model (either $50: 50$ or $80: 20$ as described in the text). For each iteration of the model $\boldsymbol{b}=\boldsymbol{b}+1$ and $\boldsymbol{a}=\boldsymbol{a}-1$-that is, there is replacement of one true negative with a false positive result. For each iteration of the model the parameters given below are calculated.

Observed probability $P_{o}=\frac{a+d}{i}$

Expected probability $P e=\frac{\left[\frac{g}{i}+\frac{h}{i}\right]}{i}$

$$
\kappa=\frac{P o-P e}{1-P e}
$$

Standard error of $\kappa \quad s e=\sqrt{\frac{P o \cdot(1-P o)}{i .(1-P e)^{2}}}$

$95 \% \mathrm{CI}$

$$
C I=\kappa \pm\left(1.96^{\star} \text { s.e. } \mathrm{\kappa}\right)
$$

PPV of a malignant $\quad P P V=\frac{d}{d+b}$
Department of

Pathology,

Royal Victoria

Hospital,

Grosvenor Road,

Belfast BT12 6BA

W G McCluggage

H Bharucha

Department of

Haematology

D Hull

E Mayne

The Queen's

University of Belfast

H Bharucha

\section{Department of}

Haematology,

St Mary's Hospital

Medical School,

Imperial College of

Science, Technology

and Medicine, London

S N Wickramasinghe

Correspondence to:

Dr W G McCluggage.

Accepted for publication 21 March 1996

\title{
Malignant lymphoma in congenital dyserythropoietic anaemia type III
}

W G McCluggage, D Hull, E Mayne, H Bharucha, S N Wickramasinghe

\begin{abstract}
A 60 year old woman with congenital dyserythropoietic anaemia (CDA) type III developed a malignant $T$ cell lymphoma with cutaneous and widespread nodal involvement. Bone marrow aspirates showed erythroid hyperplasia and dyserythropoiesis with multinucleate erythroblasts and gigantoblasts, in keeping with CDA type III. Electron microscopy showed multinucleate erythroblasts with notably irregular nuclear outlines and
\end{abstract}

intranuclear clefts. The development of malignant lymphoma in this patient, together with a documented high prevalence of monoclonal gammopathy and multiple myeloma and a single case of Hodgkin's disease, may indicate an increased incidence of lymphoproliferative disease in CDA type III.

(F Clin Pathol 1996;49:599-602)

Keywords: congenital dyserythropoietic anaemia type III, malignant lymphoma, electron microscopy. 
The congenital dyserythropoietic anaemias (CDA) are a group of rare, hereditary disorders characterised by the association of refractory anaemia with multinuclearity and other abnormalities of erythroid precursors in the bone marrow. The clinical course is generally benign, although secondary haemochromatosis may develop. The first case of CDA was reported by Wolff and Van Hofe ${ }^{1}$ in 1951, who described a family with "familial erythroid multinuclearity". CDA was subsequently classified into three major types by Heimpel and Wendt ${ }^{2}$ in 1968: type I, macrocytosis with bone marrow megaloblastoid changes and internuclear chromatin bridges; type II, normocytosis with binucleate and multinucleate marrow erythroblasts and positive acidified serum lysis (Ham's) test; type III, macrocytosis with erythroblast multinuclearity and the formation of gigantoblasts. Cases not fulfilling criteria for type I, II or III have been described subsequently. Recently, a high prevalence of monoclonal gammopathy of undetermined significance and multiple myeloma has been observed in a family with CDA type III. ${ }^{3}$ In addition, there has been a single report of Hodgkin's disease in this condition. ${ }^{4}$ Here, we describe a patient with long standing CDA type III who developed a malignant $T$ cell lymphoma. Bone marrow aspirate findings and ultrastructural features of erythroid precursors are presented. There may be an increased incidence of lymphoproliferative disease in CDA type III.

\section{Case report}

A 43 year old woman was found to be anaemic in 1976 following a routine blood test. At this time the results of a blood count were as follows: haemoglobin, $7.6 \mathrm{~g} / \mathrm{dl}$; red blood cell count, $2.2 \times 10^{12} / 1$; packed cell volume, 0.23 ; mean corpuscular volume, $106 \mathrm{fl}$; mean corpuscular haemoglobin, 35 pg; mean corpuscular haemoglobin concentration, $33 \mathrm{~g} / \mathrm{dl}$; reticulocytes, $1 \times 10^{12} / 1$; white blood cell count, $4.3 \times 10^{9} / 1$; platelets, $175 \times 10^{9} / 1$. The patient did not drink alcohol and was not taking medication. There was no family history of anaemia. No lymphadenopathy or splenomegaly was found on clinical examination. Serum vitamin $\mathrm{B} 12$ and serum folate were within the normal range. Ham's acidified serum haemolysis test was negative. A bone marrow aspirate was obtained from the iliac crest, the features of which are described later. On the basis of haematological, biochemical and bone marrow aspirate findings, a diagnosis of CDA type III was reached. Several siblings were investigated and had normal blood counts and blood films.

The patient remained well until December 1993 when she developed several raised indurated lesions on the scalp, the histology of which are described later. At that time there was no peripheral lymphadenopathy. A computed tomography (CT) scan of mediastinum and abdomen was normal, and a bone marrow aspirate and trephine biopsy specimen revealed no evidence of lymphomatous infiltration. The patient was treated with local radiotherapy.
One year later, in December 1994, the patient developed generalised lymphadenopathy with numerous palpable nodes and CT evidence of mediastinal and pulmonary involvement. A bone marrow aspirate and trephine biopsy specimen again revealed noc evidence of lymphomatous infiltration. Lymph node biopsy was performed, the histology of which is described later. Following this, the patient was treated with combination chemo-은 therapy with an initial good response. How ever, several months later she developed widespread lymphadenopathy which did not? respond to treatment. She died in December 1995.

\section{Methods}

Bone marrow smears were stained with May $-\overrightarrow{\vec{\omega}}$ Grunwald-Giemsa. Trephine biopsy specimens were processed in plastic using the epoxy resin? Polarbed 12 and were stained with haematoxy-i lin and eosin. The excised skin nodules and lymph node were fixed in formalin, routinely processed in paraffin wax and stained with haematoxylin and eosin. Immunohistochemi-근 cal staining of tissue sections was performed $\vec{c}$ using a standard Streptavidin biotin peroxidase method (Dako, High Wycombe, UK). Sections were stained with the following antibodies: LCA (leucocyte common antigen, CD45, monoclonal), UCHL1 (CD45RO, mono-O clonal), CD3 (monoclonal), L26 (CD20, monoclonal), CD15 (monoclonal), and CD30 (Ber-H2, monoclonal) (all from Dako). Immu-ণัष nostaining was carried out using appropriate positive and negative controls. Electron micro-o응 scopy was performed on plastic embedded bone marrow trephine biopsy tissue. Ultrathin sections were stained with uranyl acetate and lead citrate.

\section{Results}

BONE MARROW ASPIRATES

Bone marrow aspirates taken in 1976, 1993 and 1994 showed identical features. The cellu-음. larity of marrow fragments was normal. There N was evidence of erythroid hyperplasia, the myeloid:erythroid ratio being reduced to 0.78 . N A significant proportion of basophilic and $\mathrm{\omega}$ polychromatic erythroblasts showed dyseryth-루 ropoietic features. Four percent of erythroblasts were binucleate (normal $<0.57 \%$ ) and $\cong$ $1.1 \%$ had three to eight nuclei (fig 1A). Individual nuclei within binucleate or multinucleate erythroblasts were sometimes unequal in size and were occasionally different in shape or $\mathbb{\mathbb { D }}$ staining characteristics. Other dyserythropoi- $\frac{\Omega}{\sigma}$ etic features included Howell-Jolly bodies in $0.65 \%$ of erythroblasts (normal $<0.4 \%$ ), notably irregular nuclear outlines in $1.42 \%$ of mononuclear erythroblasts (normal $<0.55 \%$ ) $\frac{\overline{0}}{7}$ and coarse basophilic stippling of the cytoplasm. Rare giant mononuclear erythroblasts were present. Internuclear chromatin bridges (a characteristic finding in CDA type I) were not present. Some macrophages contained phagocytosed erythroblasts. 
ELECTRON MICROSCOPY

Ultrastructural examination of bone marrow showed many binucleate and multinucleate erythroblasts (fig 1B). Sometimes, individual nuclei in the same erythroblast differed in size and shape. Nuclear outlines were irregular, some nuclei containing deep clefts. In some areas there was loss of nuclear membrane material. Several nuclei were undergoing karyorrhexis. No myelin figures or other distinctive cytoplasmic features were observed.

\section{PATHOLOGY}

Histology of the scalp lesions showed unremarkable surface stratified squamous epithelium. Beneath a narrow Grenz zone, a dense infiltrate of lymphoid cells extended from the superficial dermis into subcutaneous adipose tissue (fig 2). The infiltrate largely consisted of mononuclear cells with vesicular nuclei, prominent nucleoli, irregular nuclear outlines, and a moderate amount of cytoplasm. Immunohistochemistry showed diffuse strong posi-
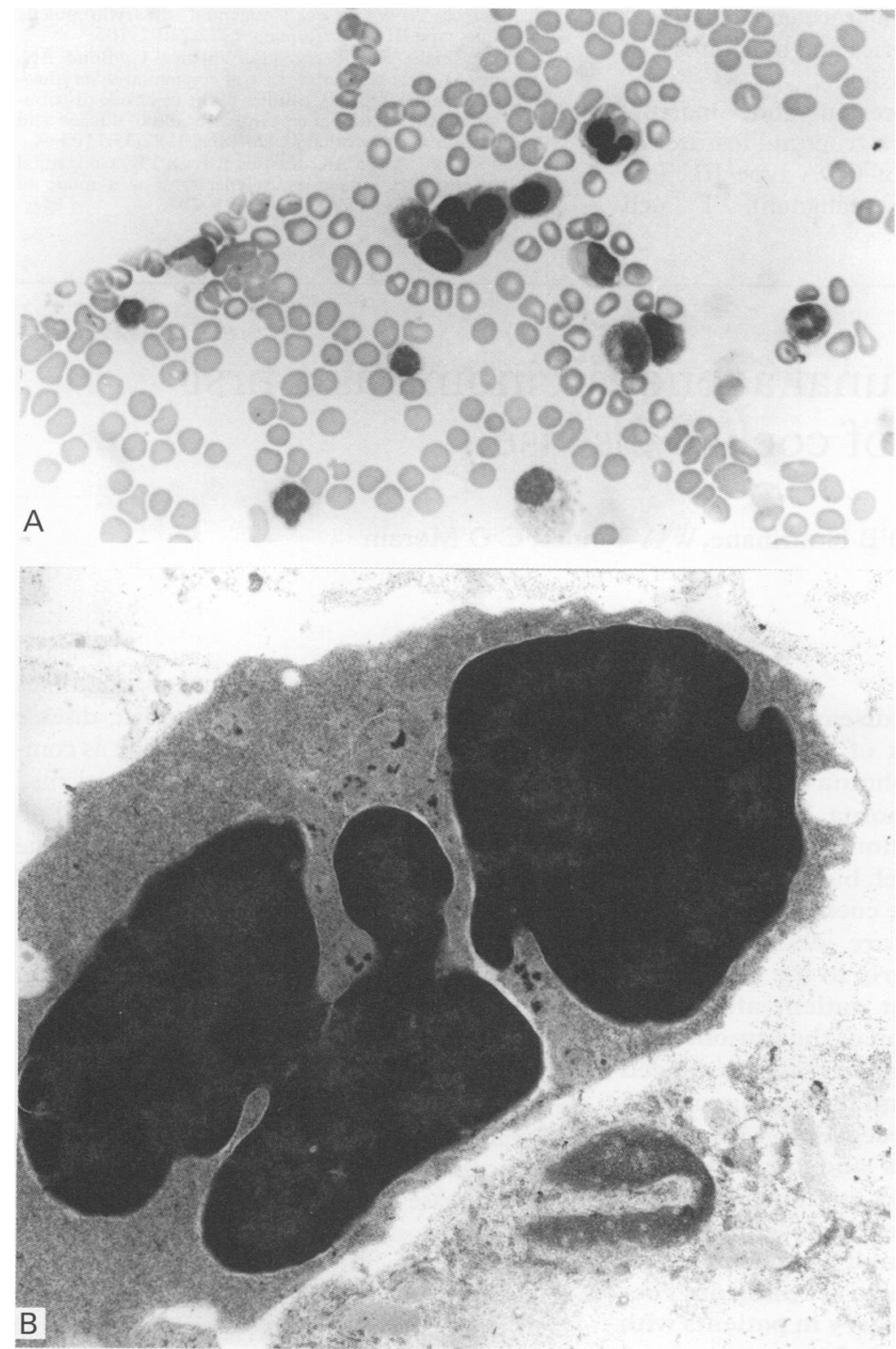

Figure 1 (A) Bone marrow aspirate from 1976 showing a multinucleate erythroblast with five nuclei. (B) Electron microscopy showing a multinucleate erythroblast with irregular nuclear outlines and intranuclear cleft formation (original magnification, $\times 15$ 000).

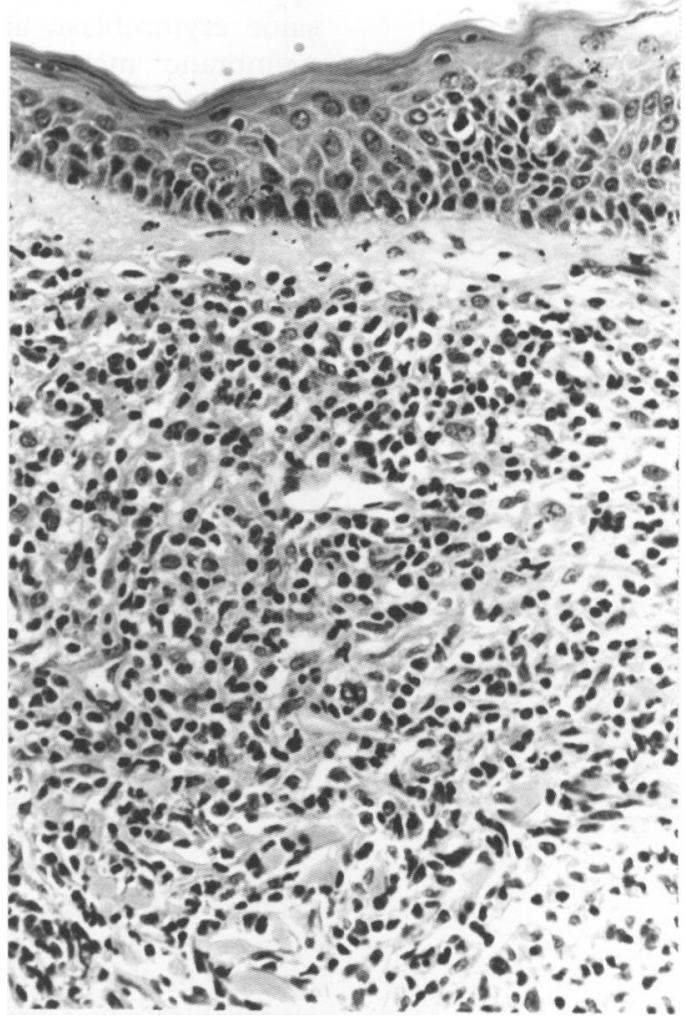

Figure 2 Skin biopsy specimen showing infiltration by malignant lymphoma.

tive staining with LCA and T cell markers UCHL1 and CD3 (fig 2). There was little or no staining with the B cell marker L26 or with CD15 and CD30. The histological diagnosis was high grade, non-Hodgkin's, $\mathrm{T}$ cell lymphoma. The excised lymph node was infiltrated by cells identical with those in the skin lesions. Immunohistochemistry confirmed nodal involvement by $\mathrm{T}$ cell lymphoma.

\section{Discussion}

CDA type III is a rare disorder of erythropoiesis, characterised by macrocytosis and the presence in the bone marrow of giant multinucleate erythroblasts (gigantoblasts) with up to 10 nuclear masses per cell, in the absence of vitamin B12 or folate deficiency. Giant mononuclear erythroblasts may also be present and erythroblasts may exhibit basophilic stippling of the cytoplasm and karyorrhexis. Granulocyte precursors and megakaryocytes generally exhibit a normal morphology although electron microscopy may reveal abnormalities in megakaryocytes. ${ }^{5}$ The disease is usually indolent with mild to moderate anaemia, sometimes with episodes of jaundice and fatigue due to haemolysis. At least two different patterns of inheritance have been documented, some families exhibiting an autosomal dominant pattern $^{16}$ while, in other cases relatives of affected individuals have been haematologically normal. ${ }^{78}$ The pattern of inheritance in the latter group may be autosomal recessive.

Ultrastructural abnormalities have been described in mononuclear and multinucleate erythroblasts in CDA type III. ${ }^{58}$ These have included intranuclear clefts, grossly disorgan- 
ised and karyorrhectic nuclei, differences in the appearance of individual nuclei within the same erythroblast, and focal loss of nuclear membrane material. Mitochondrial abnormalities, autophagic vacuoles, extensive myelinisation of the nuclear membrane, and the presence of intracytoplasmic myelin figures have also been reported.

CDA type III has been described in only a few families and individuals. ${ }^{134679}$ Sandstrom $e t a l^{\beta}$ in 1994 in an extensive investigation in a known family with CDA type III $^{5}$ observed a high prevalence of monoclonal gammopathy of undetermined significance and multiple myeloma. The authors speculated that genes for the development of monoclonal gammopathy/myeloma and CDA type III may be linked. Prior to this, Byrnes et al in 1980 described an individual who had been treated by radiotherapy for Hodgkin's disease and was found to have abnormalities of erythroblasts on a bone marrow aspirate. The abnormalities were initially interpreted as being radiotherapy induced but review of a bone marrow aspirate taken 18 years earlier revealed identical changes in the red cell series and a diagnosis of CDA type III was established.

In conclusion, we present bone marrow aspirate findings and ultrastructural features of erythroblasts in a case of CDA type III. The patient developed a malignant $T$ cell lymphoma with cutaneous and widespread nodal involvement. This case, in conjunction with previous studies, ${ }^{34}$ suggests that there may be an increased risk of development of lymphoproliferative disease in CDA type III. The mechanism of this association is not clear but it may be that genes for the development of $\frac{\rho}{5}$ CDA type III are linked to those which control 0 lymphoid proliferation or development.

1 Wolff JA, Von Hofe FH. Familial erythroid multinuclearity. Blood 1951;6:1274-83.

2 Heimpel H, Wendt F. Congenital dyserythropoietic anaemia with karyorrhexis and multinuclearity of erythroblasts. with karyorrhexis and multin

3 Sandstrom H, Wahlin A, Eriksson M, Bergstrom I, Wickramasinghe SN. Intravascular haemolysis and increased $\mathbb{\Phi}$ prevalence of myeloma and monoclonal gammopathy in congenital dyserythropoietic anaemia, type III. Eur $\mathcal{F} \mathrm{Hae}-\mathrm{n}$ matol 1994;52:42-6.

4 Byrnes RK, Dhru R, Brady AM, Galen WP, Hopper B. $\vec{O}$ Congenital dyserythropoietic anaemia in treated Hodgkin's disease [letter]. Hum Pathol 1980;5:485-6.

Wickramasinghe SN, Wahlin A, Anstee D, Parsons SF, Stopps G, Bergstrom I, et al. Observations on two members of the Swedish family with congenital dyserythropoietic anaemia, type III. Eur f Haematol 1993;50:213-21. A

6 Bergstrom I, Jacobsson L. Hereditary benign erythroreticulosis. Blood 1962;19:296-303.

7 Goudsmit R, Beckers D, DeBruijne JL, Engelfriet CP, James J, Morselt AFW, et al. Congenital dyserythropoietic $\bullet$ $\mathrm{J}$, Morselt AFW, et al. Congenital dyserythro

8 Wickramasinghe SN, Parry TE, Williams C, Bond AN, 은 Hughes $M$, Crook S. A new case of congenital dyserythropoietic anaemia, type III: Studies of the cell cycle distribu- $c$ tion and ultrastructure of erythroblasts and of nucleic acid synthesis in marrow cells. F Clin Pathol 1982;35:1103-9.

9 Choudhry VP, Saraya AK, Kasturi J, Rath PK. Congenital dyserythropoietic anaemias: splenectomy as a mode of therapy. Acta Haematol 1981;66:195-201.

\title{
Duodeno-jejunal adenocarcinoma as a first presentation of coeliac disease
}

\author{
D J L MacGowan, D O'B Hourihane, W A Tanner, C O'Morain
}

\begin{abstract}
Department of Gastroenterology, Meath/Adelaide and St James's Hospitals, Trinity College, Dublin, Ireland C O'Morain

Department of Surgery W A Tanner

Department of Pathology

D J L MacGowan

D O'B Hourihane

Correspondence to: Professor Colm O'Morain, Department of Gastroenterology, The Meath Hospital Heytesbury Street, Dublin 2,

Ireland.

Abstract

Long standing coeliac disease is associated with an increased risk of malignancy, not only of intestinal lymphoma but also small intestinal adenocarcinoma. Two patients whose initial presentation was adenocarcinoma of the small bowel, but who were subsequently found to have coeliac disease after Whipple's resection, are described. The diagnosis was made early in the postoperative period in the first patient after close histological examination of the tumour-free mucosal margins. This patient was placed on a gluten-free diet and had an uncomplicated postoperative recovery with rapid weight gain. Diagnosis and dietary intervention in the second patient was very delayed and resulted in the development of severe malabsorption and weight loss. This illustrates the importance of ruling out coeliac disease prior to surgery in patients with small intestinal malignancies.

$(\mathcal{C}$ Clin Pathol 1996;49:602-604)
\end{abstract}

Keywords: small bowel, adenocarcinoma, coeliac disease.
Small intestinal adenocarcinoma is 82 times more common in patients with coeliac disease $\frac{D}{0}$ than in the normal population, making it as common a tumour as colon cancer in these patients. ${ }^{1}$ o This relation has been reported in a total of $40 \mathrm{~N}$ patients. ${ }^{1-8}$ In the 19 patients for whom adequate information is available, ${ }^{235-8}$ the presentation of 0 malignancy was preceded by symptoms ofo malabsorption and usually a longstanding history $₫$ of coeliac disease. This is therefore the first report ${ }^{+}$ describing two patients in whom the diagnosis of small bowel adenocarcinoma was not preceded $\stackrel{\circ}{\mathrm{\sigma}}$ by a history of malabsorption. Both patients $\stackrel{\sim}{\mathbb{D}}$ developed symptoms of malabsorption after sur- $\frac{\alpha}{9}$ gery, raising a possible mechanism of latent coeliac disease being unmasked by surgery. Latento̊ coeliac disease (high intraepithelial lymphocyte count with positive $\alpha$-gliadin antibodies) is ano을 increasingly recognised entity, ${ }^{9}$ but a second factor seems to drive this minimal enteropathy from latent to clinically overt disease. Following gastrectomy or upper gastrointestinal surgery, the bowel may be transiently hyperpermeable on reintroduction of oral intake and the intraluminal 